\title{
Nay or Nyay 3: Epistemic Utility of Shadow of Ignorance that Obscures Mental Images and Associated States
}

\author{
Mahendra Kumar Jain \\ University of Delaware
}

\author{
Rafael Apitz-Castro \\ Academia Nacional de Medicina
}

\begin{abstract}
Survival of organisms requires response to sense inputs and feedback. An intuitive picture or mental image of reality from sense experience is apparently structured from orthogonal and independent sense inputs in association with past memories. Such images are not necessarily visual but may have such qualities. Play of mind with information in mental images processed by molecular actors is guided with rules of reality to assure non-contradictory outcome of actions for successful behaviors (Rogers and Jain 1993). Such functions have evolved to find food before becoming food, and fight or flight when in doubt. Shadows of ignorance that obscure mental images and associated states also have epistemic utility. Their success depends on learning from trials and errors to compensate for incomplete information and uncertainties with consideration of what could go wrong. Outcomes are also influenced by games played on mind by illusions, mistakes, surprises, ignorance. Loss of time, energy, and opportunity associated with ignored or misinterpreted information threaten survival. Equivocation, cynicism, wild goose chase, and vicious circle of sterile ideas encourage contradictory or inconsistent interpretations that compromise outcomes. Throughout the human history, ignorance of horsemen of apocalypse has unleashed havocs, perpetuated wars, epidemics, wrongful medical treatments, and economic disasters. What used to be crisis of ignorance has now become crisis of unintended consequences of inventions and other forms of knowledge. Ignorance of experts and head of states as horsemen of darkness brings misery to countless innocents. In Nay formalism, knowledge (gyan) is what knows with certainty (either as true or false). Identifying uncertainties introduces doubt (syad) in what one knows. For finding ones way around lack of relevant evidence (agyan), wisdom lies in recognition of ignorance and identifies problem to seek solution. Note that these states for propositions are not necessarily related by binary negations.
\end{abstract}

Keywords: epistemic value, mental images, ignorance, doubt, certainty, apocalypse, misrepresentation, misinterpretation

Certain features of the central nervous system (CNS) for the functions of mind are reasonably well understood. CNS is a network of brain and associated organs to cognize discrete objects and concerns perceived from sense experience. Species specific architecture of organs is evolutionarily determined. Brain is organized with genetically inherited instructions to communicate with other organs (Swanson 2003), and their CNS functions are established under epigenetic influences during the development (Cabej 2012). Organisms at

Mahendra Kumar Jain, emeritus professor, Department of Chemistry and Biochemistry, University of Delaware, USA; main research fields: 2D-Interfacial Catalysis and Logic of Doubt in Evidence-Based Inference. Email: mkjain@udel.edu.

Rafael Apitz-Castro, M.D., retired, Academia Nacional de Medicina, Venezuela; main research fields: Biochemistry, Biophysics and Hematology Relevant to Human Physiology and Philosophy of Human Behaviors. Email: rapitz@gmail.com. 
birth are instinctively aware of their survival needs, and their prenatal functions are further coordinated and optimized with inputs from the environment that is likely to provide for its needs. For such purposes, sense inputs are interpreted and outputs are communicated to neuromuscular system for rapid motor response and feedback, and also to neuroendocrine system for somewhat slower metabolic response, including energy supply and storage of information. Through such functions, the mind learns to project potential liabilities of unknowns.

Sense organs for physical and chemical stimuli (touch, taste, odor, light, sound) are windows for the external reality in real time and space. Interactions with the environment are also facilitated by other sensors, such as shifting of the center of gravity to position and balance body movements. Specific receptors on internal organs similarly mediate response to pain, hunger, thirst, osmotic changes, temperature, regulators, signals, and messages. Slower and longer lasting effects of stimuli are mediated via coupled trans-membrane receptors that regulate cytoplasmic functions. Information about intensity and gradients of stimuli received by sense receptors for rapid response is coded as sequence of electrical signals and transmitted through bundles of nerve fibers to specific regions of the brain. Regions of brain have specific functions (Swanson 2003). Its layered structure is nearly developed in a newborn, and additional neural circuits with migration of pre-natal neurons are laid out later for specific functions. During the first 18 months, the number of synapses increases more than ten-fold, and then decreases by about $40 \%$ through the rest of life. Such synaptic plasticity is believed to upgrade and strengthen neural circuits used during the postnatal development, and those not used become dormant or are lost.

There is emerging consensus that the rapid information processing functions of CNS are mediated by electrical changes in circuits of neurons interconnected via synapses on their axon and dendrite extensions (Kandel 2006). Trans-membrane gradients of ions and their relative permeability determine the membrane potential. Neurotransmitters and membrane potential regulate ion channels and in turn control threshold for excitability, frequency, duration, and rate of propagation of action potentials for transmission of information. Signatures of electrical activity in regions of brain are diagnostic for mental functions. Nuclear magnetic resonance images also show accumulated metabolic changes in regions of brain associated with psychological and disease states. Loss of motor control in Parkinson's disease is associated with loss of only 20,000 neurons in a certain region of the brain. Human brain has 80 billion glial cells, and as many neurons interconnected possibly through 10,000 synapses for each neuron (Herculano-Haouzel 2009). These provide more than 100 trillion components for neural circuits.

Nature and nurture together program CNS to interpret and respond to sense inputs. Attributes of individual mind and identity develop with skills and behaviors that seek significance of sense experiences and meaning of mental images. Architecture of neural circuits for information processing functions remains to be conceptualized. Ancient suggestions invoked duality of brain as a part of worldly physical body and mind as part of the other-worldly omniscience. Mind as engine, machine, electronic circuit, or binary digital computer also failed to provide insight. Our focus below is on word communication that requires processing of sense information in real-time, and thus also provides insights into utility of ignorance.

Mimicry, vocalization, and speech, including slip of tongue or tongue tied, are neuromuscular responses of CNS. Quality of vocalization is a marker of sexual differentiation, health, virility, as well as cognitive dissonance. Words communicate awareness of an object or concern abstracted as a discretized part of experience. Symbolic languages permit descriptions that anchor meaningful parts of mental images to communicate awareness of an experience to share concerns and deal with uncertainty and ignorance. Rule 
bound descriptions of the observed and measured parts of an object evoke cognition of its content and context to infer behaviors. Perceptions of meaning and significance of sense experiences guide choices and decisions for actions. Thus, word representations permit ready reference of objects and relations for concept formation.

Fleeting mental images may derive in real time or emerge after the fact from flickers of ever changing sense experiences (Pinker 1997; Shepard 1978). Some persist and most dissolve away into oblivion. Variations in the content, medium, detail, and other features of mental images are likely to be as varied as individual perceptions and motives. Words anchor significant parts of mental images, and word constructs capture relations between the parts. Word descriptions are meant to elicit comparable mental images in the listener and the speaker, and thus communicate information about sense experience. Convention bound languages further facilitate manipulation, deliberation, and discourse for scrutiny, validation, and evaluation of implications, consequences, meaning, and significance of shared experiences. Written forms liberate thought from extraneous influences. Assertions and propositions that track rules of reality lead to inferences that are likely to be affirmed by independent evidence. Insights and ideas rooted in shared realities address concern and resolve conflict. Their representations as part of empirical knowledge remain deficient and incomplete (Rescher 2009). Measure of success of shared representations is utility of outcome, and their shortcomings are addressed by trial and error (Feyerabend 1975). Words devoid of real content and context generate sterile images that do little more than evoke ill-defined emotions and feelings.

It is remarkable that word-strings as one-dimensional renditions of mental images of multidimensional experience of a speaker evoke comparable awareness, cognition and perception in the mind of a listener. Such communication builds on the tension and dynamics at the boundary of what is said and what remains unsaid. In words of Thomas Pynchon: Ignorance is not just a blank space on a person's mental map. It has contours and coherence, and all I know rules of operation as well. So as a corollary to writing about what we know, maybe we should add getting familiar with our ignorance. Epistemic value of identified ignorance is not only in what is shadowed but also what casts the shadow. Paradoxically, awareness of what we do not know makes us want to know more. Also more we know we are likely to see more and want to know more.

Certainty against shadows of ignorance is an experienced quality of knowledge (Dovring 1998). What one knows lies against the backdrop of the awareness of what else one knows, what else is needed, and whether or not it exists. Processing of information and evidence for learning, understanding and discovery of it is proceed along its boundary with it is not. Such epistemic nuances are communicated with subjunctives as in to not doubt that..., to be certain that..., to think that..., to not deny that..., it's true that..., and other expressions that trigger indicative mood. Perceived ignorance at or beyond the known boundaries is qualified as to doubt that..., it is doubtful that..., it's unlikely that..., it's uncertain that..., it's possible that..., not to believe that..., it's not certain that..., to not be convinced that..., to not be sure that..., to not seem that..., to not think that..., to not suppose that..., it may be that..., to deny that..., it's not true that..., it's not certain that..., to not imagine that..., to suspect that..., surprisingly it is that... Expressed acuity of sense experience encourages exploration of objects and concerns in fiction and stream of consciousness that resonate with feelings and emotions.

Absolute certainty about all-inclusive it is is the realm of omniscience. Essence of science is measured certainty about small parts of the world. Reasoning for epistemic certainty about it is relates to what it is not or is unlikely on the basis of available evidence. Methods of science seek incremental certainty for it is in relation to what remains as likely but is not yet resolved with independent evidence. Each new discovery uncovers a part of what was not known before, and also brings to light its relations to all that is known. It increases 
awareness of what is still unknown. Like new shoots on a vine, such knowledge incrementally fills the gaps in the mental image of the underlying reality. It unleashes forces that continually influence quality of life. Their unintended consequences influence the willing and unwilling participants who fail to adapt to changes (Jain 1998; 2001; 2006).

Science is at its best when faced with the challenge of the unknown that is amenable for asking meaningful questions. A recent thought-proving primer (Firestein 2012) asserts that science is driven by such ignorance. It is for appreciation of good ignorance by undergraduates and citizen scientists. It builds on insights of other scientists, and I am sure similar views are held by all who labor in the trenches of discovery. My take for what follows is that ignorance is a necessary part of epistemology to establish knowledge. It is not about paradigm shifts that redirect thought at critical historical junctures (Kuhn 1962), nor is it about building an all-purpose road where meandering trails existed before. It is about trail blazers who work with little more than a mental image of the terrain. It is an Alice in Wonderland kind of world to be fathomed by wobbling Through the Looking Glass that separates the known from unknowns including ignorance of not knowing where one stands and where to the next step.

Conundrum of doubt and ignorance (Rothman and Sudarshan 1998) can be disconcerting. Some quit in frustration (Schwartz 2008). For those who persevere (Feynman 1984), the scientist has a lot of experience with ignorance and doubt and uncertainty, and this experience is of very great importance. When a scientist doesn't know the answer to a problem, he is ignorant. When he has a hunch as to what the result is, he is uncertain. And when he is pretty darned sure of what the result is going to be, he is in some doubt. We have found it of paramount importance that in order to progress we must recognize the ignorance and leave room for doubt. Scientific knowledge is a body of statements of varying degrees of certainty-some most unsure, some nearly sure, none absolutely certain. Monod generalizes: science is nourished by restlessness, anxiety, dissatisfaction, and agony of mind. There are good reasons to sweat over the unknowns. An educated guess about what it is that one is after is the beginning to formulate questions, select suitable methods and tools for observation and measurement, design controls, interpret results, spot serendipity to turn surprises into opportunity, to see if something is wrong, and whatever comes next. Quality of ignorance in a fishing expedition by trial and error is not the same as in random walk of drunken sailor looking for dark cat in dark room without knowing if it is there.

Awareness of what may be shadowed by ignorance is an uncommon sense that turns hunches and gut feelings into ideas and actions by discarding make-beliefs and sterile dead ends. Information and understanding abstracted from observable and measurable parts of world provide insights into causal and hierarchical relations that uncover ignorance of what remains unresolved. Perception of certainty built on meaningful parts of known world empowers search for liabilities to nourish futuristic visions and musings of what it could be (Maddox 1998). Mentors motivate budding scientists to see beyond the obvious, provide skills to spot realities in mental images, and persevere to meaningfully entertain doubt and turn ignorance into opportunities for lifelong pursuit of hypotheses for viable ideas, and much more. A prepared mind follows leads and benefits from lucky breaks for aha moments. Such skills are also useful to ward off buzz of bad ignorance encountered not only in cocktail banter but in pseudo-intellectual coverage of politics, faith, economics, health, nutrition, medicine, global climate change, and other life and death issues.

Enviable success of science comes from methods of trial and error. The process appears and is chaotic. Science emerges stronger after each confrontation as its understanding thrives on scrutiny. Certainty about an 
inference increases with each reevaluation with independent evidence, criteria, or whatever else may surface as concerns. There is no definite end-point for this open-ended search where incremental certainty follows from ignorance uncovered. Their epistemic utility emerges as each doubt that is resolved leads to the next, and also from insights from unintended consequences, surprises, and serendipity.

Certainty with conservation of information is about whether or not something exists as the starting point to balance what remains. Classical binary logics for deduction use complementation of not-true as false with unknowns excluded and ignored. It permits closure of relations in Boolean algebra (Jain 2011). Excluded middle is expressed as fractional probabilities of crisp logic states (Smithson 1988), or as a set of fuzzy logic states. Bayesian theorem re-considers probabilities with emerging evidence (Bernardo and Smith 1994; Bovens and Hartmann 2003; Williamson 2010). Evidence- and outcome-based methods practiced in medicine, law, education, administration, and other complex systems have the caveat that correlation is not causation. Skills are also required to continually ask questions to uncover shades of ignorance (Fiengo 2007), and to identify and evaluate emerging surprises (Gross 2010; Halpern 2003; Hammond 1996; Lemons 1996; Trinder and Reynolds 2000; Zimmerman 2008). It appears that search for a valid inference may follow a convergent polynomial where certainty increases by successive resolution of liabilities in the higher order terms.

Prior knowledge as well as memories and beliefs as part of a mental image facilitates judgment and constrains interpretations. Discourse in science moves forward with I do not see anything obviously wrong, but how about this or that. It builds awareness of what remains (Malewski and Jaramillo 2011). Einstein's quip that god does not play dice did not discourage pursuit of quantum mechanics which opened up whole new phenomenal world of subatomic particles. Twentieth century physics has successfully negotiated such serious challenges to rework its founding assumptions. The Newtonian universe is extended by Einstein's relativity but by constraining velocity of light and Planck's constant as the universal constants. Heisenberg's uncertainty principle showed that the Planck's constant places a limit on the resolving power of measurements on quantum objects, and thus restricts their descriptions in terms of the rules of classical mechanics. The incompleteness theorem places a limit on the analytical provability (Godel 1931; Smullyan 1987) with the demonstration that in mathematical systems a false statement is not provable, and there also exist statements that are true but not provable. Conservation of mass, energy, and information are dictates of reality that place thermodynamic limits on world happenings, and also rule out Maxwell's demon, perpetual motion, and omniscience as contradictions to reality. Understanding genetic code provides a place for human in the hierarchy of biological diversity that rules out notions of race and chosen people (Jain 1998; Wade 2006).

Faiths, fictions, and secular philosophies consider ignorance and doubt as perpetual human condition, but do not offer resolution. The wise of the old also noted that the learned, pseudo-intellectuals, and bookworms lean on crutches of words rather than commonsense rooted in reality interpreted with strength of thought (Hazlitt 1824). Ugly and insidious kind of ignorance is perpetuated with sophistry of words for mass deception and distraction (Meyer 2010) by lobbies for asbestos, tobacco, sugar, genetically modified foods, and scores of other products are as bad as real weapons of mass destruction (WMD). They follow a common script: Products are introduced in the name of progress, sold with promise of jobs, continued with claims of no-evidence for harmful effect, and then benefits outweigh the cost or demonstrated consequences are not significant. It is all cost of doing business and then moves out before liabilities make it unprofitable to continue. Strategies to perpetuate ignorance in politics, faith, war, and love include illusions, paradoxes, self-reference, unverifiable lies covered by astro-turfing and psy-ops. Some are swayed. A smoker with a Ph.D. degree in physics told me 
that there is no evidence that smoking is harmful to him. Of course he is right except that it will be too late for him to find out if and when harm is done to him which happened few years later. Ignorance is more than just being uninformed.

Search by trial and error is kept on track with open inquiry backed by uncompromising honesty and healthy skepticism. It is misled by dishonesty, authority, secrecy, fog of misinformation, premature, and ad hoc beliefs (Cornwell 2003; Hook 2002). God complex and tendency to spin tales to fit agenda also perpetuate vicious cycle of self-reference, as in the liar's paradox, or in the life or death of Shrodinger's cat on the basis of certainty about past events, or with the assertion that human brain is the most wonderful thing. In fact, much of the ignorance emanates from paradoxes resulting from extrapolation of self-referential assertions. Consider mutually contradictory forms of ad hoc omniscience or perfect knowledge (Armstrong 1993; Dawkins 2006) presented as a binary equivocation of it is (existence) against it is not (nonexistence). Objects of such faith are often justified by Pascal's wager with a payoff just in case it exists, and little harm done if it turns out that it does not. It matters little to the faithful whether there is no evidence for the object of faith, or it is inconsistent or contradictory, or if the consequences of its presence versus absence are indistinguishable. Agnostics somehow feel that it is necessary to have evidence for it is not, even if there is no evidence for either it or for is. Atheists see little reason to be concerned unless an object is affirmed by independent evidence. Also it is not possible to reason unless a description elicits awareness for a mental image.

A valid inference follows from assertions affirmed by evidence (Jain 2011). Assertion of its converse requires independent evidence. Also lack of evidence for an assertion is also not the evidence for its negation. In binary deduction, a proposition may be judged true on the basis of certain criteria. If not so, it is judged not-true, and worse still as false. If it walks like a duck and it quacks like a duck, it is a duck is still such a deduction no matter how many other attributes of duck are enumerated. An ancient Indian parable of elephant and six blind men aptly illustrates the inference process. Each describes their touch and feel experience with analogies to rope, brush, hard curved stick, pillar, flexible tree-trunk, or a wall. As a good parable, the rest is a thought experiment. For example, consider the option if they have seen or not seen an elephant, or only heard about it. How would they rationalize the analogies in terms of what they knew about elephants? For an inkling of the discovery process, consider if nobody has ever seen the beast before.

In the logic of inference, true and false assertions are orthogonal to be independently affirmed to avoid self-reference. It makes it different from binary deduction, tree-pruning, or pattern recognition. Mental images may be structured from such orthogonal assertions affirmed by sense evidence. It appears that two to eight independent inputs are typically incorporated into a mental image for real-time manipulation. Physiology of vocal cord and resonance cavity is such that it generates four to seven sounds distinguishable by human ear, and such vowels can be mixed with tongue movements to give sound to alphabets. Most commonly used words also have two to eight characters. Similar numerical limits also hold for the number of words in a sentence or sound bite, or the interactions in anecdotes and short stories, or the key characters in a novel, or the variables and constants in a polynomial, or the number of figures and table in a scientific paper.

As a step towards a theory of mind, consider inference output as the solution to a matrix of orthogonal relations in a mental image (Jain 2011). The tradition that gave the elephant parable also gave a minimum matrix for inference. This syllogism for evidence-based inference builds the proposition it exists for an object from a set of three orthogonal criteria: Sense inputs for the observed and measured attributes (A); awareness of sense inputs as the basis for description (D); and the ability of mind to abstract and evaluate differing 
consequences (C) for example of the presence versus absence of the object. The proposition it exists is thus inferred from attributes that describe and demonstrate consequences of an object. Eight logic states are possible with evidence for none, one, two, or all the three criteria (or $2^{n}$ states with $n$ criteria). Their logic space is a 3x8 matrix of truth table with 1 for affirmed and 0 for not affirmed. Additional inputs and merged matrices could accommodate more complex mental images.

Logic states are the outputs of matrices of inputs. Matrices of orthogonal inputs can also be implemented as logic circuits. A neural circuit with three or more inputs could implement binary functions as irreversible or reversible conditional gates, filters, buffers, and memories. The 3x8 matrix can also be partitioned for logically reversible controlled Toffoli gate for universal binary operators, or configured as irreversible classical binary gates with 3- or 2-inputs. A matrix with 3-input could also be configured as a qubit (quantum bit) whose quantum integrity decoheres in less than microsecond. There is no obvious reason why such matrices could not be implemented in neural circuit. If so, resulting nubits could persist for seconds and longer in analogy with mental images, and modified with additional inputs and feedback. Algorithms for processing such inference devices are likely to be different, but their limiting cases would be binary functions.

The classical interpretation of the eight logic states provides a reasonable intuitive basis for an appreciation of their epistemic utility to identify entropic liabilities. There is no reasoned proposition if there is no evidence for A, D, and C. Nothing can be said about it except that it is a state of nothingness or a null that may also be used to orient additional inputs. Negation of it does not exist also requires affirmation by independent evidence. Virtually all variations of bad ignorance whirl in vortex of such black holes. Each of the other seven states (called the Saptbhangi) has epistemic utility for reasoning in relation to affirmed assertions. The inference proposition it exists is fully supported by independent evidence for A, D, and C. Lack of evidence for one or two assertions in the other six states identifies source of ignorance with possible origins in incomplete information, evidence, or knowledge. Such states manifest as uncertainty, confusion, chaos, doubt, ambiguity, contradiction, vagueness, fuzziness, dissonance, risk, and conflict (Colyvan 2008; Firestein 2012; Gross 2010; Halpern 2003; Hammond 1996; High 2012; Lemons 1996; Roth and Ross 1990; Rothman and Sudarshan 1998; Smithson 1988; Suri and Bal 2007; Zimmerman 2008).

In short, ignorance is inability to transpose metal images to alternate universes, and with bad ignorance one remains stuck in a self-referential world. Shadows of ignorance are a necessary part of establishing certainty about what one knows and infers. A syllogism for inference with orthogonal assertions affirmed by independent evidence provides a common basis for representation of multiple logic states in Hilbert space of the orthogonal assertion vectors (Jain 2011). Many other states, including ignorance and uncertainty, could also be represented in such logic space. It also provides a basis for a theory of mind where the matrix of assertion vectors may provide a template for mental images from parallel and independent inputs from the sensory (information), speech (memory), and reasoning functions of CNS. Its output as an inference could be weighted for response and modified by feedback. Mind as an inference machine trained with inputs from the past to form memory templates could also pattern incoming inputs into mental images. Beliefs could fill gaps in such mental images to address doubt, and could also overshadow parts and relations in real time inputs.

\section{Works Cited}

Armstrong, K. History of God: The 4000 Year Quest of Judaism, Christianity and Islam. New York: Knopf, 1993. 
Bernardo, J. M. and A. F. M. Smith. Bayesian Theory. New York: Wiley, 1994.

Bovens, L. and S. Hartmann. Bayesian Epistemology. Oxford: Clarendon Press, 2003.

Cabej, N. R. Epigenetic Principles of Evolution. Amsterdam: Elsevier, 2012.

Colyvan, M. “Is Probability the Only Coherent Approach to Uncertainty?” Risk Analysis 28 (2008): 645-52.

Cornwell, J. Hitler's Scientists: Science, War and the Devil's Pact. New York: Viking, 2003.

Dawkins, R. The God Delusion. New York: Houghton-Mifflin Co., 2006.

Dovring, F. Knowledge and Ignorance; Essays on Lights and Shadows. Westport Conn.: Praeger, 1998.

Feyerabend, P. Against Method: Outline of an Anarchistic Theory of Knowledge. London: Versso Editions, 1975.

Feynman, R. P. Surely You're Joking, Mr Feynman: Adventures of a Curious Character. New York: W. W. Norton, 1984.

Fiengo, R. Asking Questions: Using Meaningful Structures to Imply Ignorance. New York: Oxford University Press, 2007.

Firestein, S. Ignorance: How It Drives Science. New York: Oxford University Press, 2012.

Gross, M. Ignorance and Surprise: Science, Society, and Ecological Design. Cambridge: The MIT Press, 2010.

Halpern, J. Y. Reasoning about Uncertainty. Cambridge: The MIT Press, 2003.

Hammond, K. R. Human Judgment and Social Policy: Irreducible Uncertainty, Inevitable Error, Unavoidable Injustice. New York: Oxford University Press, 1996.

Hazlitt, W. Table Talk (Eassay on the Ignorance of the Learned, p. 159-77, Vol. 1). London: Henry Colburn, 1824.

Herculano-Haouzel, S. “The Human Brain in Numbers: A Linearly Scaled-up Primate Brain.” Frontiers in Human Neuroscience 3 (2009): 1-11.

High, C., Kelly, A. H., and Mair, J. The Antropology of Lgnorance: An Ethnographic Approach. New York: Palgrave MacMillan, 2012. 220.

Hook, E. B. Prematurity in Science. Berkeley: University of California Press, 2002.

Jain, M. K. “Greed and Grab: Many Are Called yet Few Are Chosen.” Quarterly Review of Biology 73 (1998): 329-32.

---. "Human Natures: Genes, Cultures, and the Human Prospect (Book Review).” Quarterly Review of Biology 76 (2001): $345-6$.

---. “Is Human Nature Obsolete?” Quarterly Rev. Biol. 81 (2006): 91-92.

---. “Logic of Evidence Based Inference Propositions.” Current Science 100 (2011): 1663-72.

Kandel, E. C. In Search of Memory. New York: W. W. Norton, 2006.

Kuhn, T. The Structure of Scientific Revolutions. Chicago: Chicago University Press, 1962.

Lemons, J. E. Scientific Uncertainty and Environmental Problem Solving. Cambridge: Blackwell Science, 1996.

Maddox, J. What Remains to Be Discovered: Mapping the Secrets of the Universe, the Origin of Life, and the Future of the Human Race. New York: The Free Press, 1998.

Malewski, E. and N. Jaramillo. Epistemologies of Ignorance in Education. Charlotte: Information Age Pub., 2011.

Meyer, P. Lie Spotting: Proven Techniques to Detect Deception. New York: St. Martin’s Press, 2010.

Pinker, S. How the Mind Works. New York: W. W. Norton, 1997.

Rescher, N. Ignorance: On the Wider Implications of Deficient Knowledge. Pittsburgh: University of Pittsburgh Press, 2009.

Rogers, J. M. and M. K. Jain. “Inference and Successful Behavior.” Quarterly Rev. Biol. 68 (1993): 387-97.

Roth, M. D. and G. E. Ross. Doubting: Contemporary Perspectives on Skepticism. Dordrscht: Kluwer Academic Publishers, 1990.

Rothman, T. and G. Sudarshan. Doubt and Certainty. Reading: Helix Books, Perseus Press, 1998.

Schwartz, M. A. “The Importance of Stupidity in Scientific Research.” J. Cell Sci. 121 (2008): 1771.

Shepard, R. N. “The Mental Image.” American Psycohologist 33 (1978): 125-37.

Smithson, M. Ignorance and Uncertainty: Emerging Paradigms. New York: Springer-Verlag, 1988.

Suri, G. and H. S. Bal. A Certain Ambiguity: A Mathematical Novel. Princeton: Priceton University Press, 2007.

Swanson, L. W. Brain Architecture. Oxford: Oxford University Press, 2003.

Trinder, L. and S. E. Reynolds. Evidence-Based Practice: A Critical Appraisal. Oxford: Blackwell Science, 2000.

Wade, N. Before the Dawn: Recovering the Lost History of Our Ancestors. New York: Penguin Press, 2006.

Williamson, J. In Defence of Objective Bayesianism. New York: Oxford University Press, 2010.

Zimmerman, M. J. Living with Uncertainty: The Moral Significance of Ignorance. Cambridge: Cambridge University Press, 2008. 